\title{
Scalability of a parallel monolithic multilevel solver for poroelasticity
}

Arne Nägel and Gabriel Wittum

\begin{abstract}
This study investigates a solver for the quasi-static Biot model for soil consolidation. The scheme consists of an extrapolation scheme in time, complemented by a scalable monolithic multigrid method for solving the linear systems resulting after spatial discretisation. The key ingredient for the later is a fixed-stress inexact Uzawa smoother that has been suggested and analysed using local Fourier analysis before [8]. The work at hand investigates the parallel properties of the resulting multigrid solver. For a 3D benchmark problem with roughly 400 million degrees of freedom, scalability is demonstrated in a preliminary study on HazelHen. The presented solver framework should be seen as a prototype, and can be extended and generalized, e.g., to non-linear problems easily.
\end{abstract}

Key words: multigrid, poroelasticity, parallel computing

\section{Introduction}

As access to high-end computational systems is nowadays widely spread and computational time is available in ever increasing amounts, this sparks the desire to solve larger and larger problems in the applications. The goal of this work is to develop an efficient and scalable solver for poroelasticity, i.e., porous media flow problems considering deformations of the medium. We fo-

Arne Nägel

Goethe-Center for Scientific Computing, Goethe-Universität Frankfurt,

Kettenhofweg 139, 60325 Frankfurt,

Tel.: +49-(0)69-798-25283,

e-mail: naegel@gcsc.uni-frankfurt.de 
cus on the linear quasi-static Biot model [2]. This system is a saddle point problem. At the core is a constraint for the stresses, coupling deformations and pressure. The second component is the flow equation, which considers the deformation of the medium in the volume terms of the time derivative.

Solvers for poroelasticity (and coupled processes in general) may be subdivided into the following classes (cf. [17]).

Fully implicit schemes (or: monolithic schemes) tackle the block system resulting from the spatial discretisation as single monolithic block. Efficient solvers rely on blocking strategies, thus require access to the software infrastructure, and must be implemented with proper care.

Iterative coupling is an alternative strategy. As the name indicates, convergence is achieved by iterating back and forth between the equations for the different unknowns. This approach is more modular than monolithic schemes and is less intrusive with respect to software infrastructure. For poroelasticity different algorithms have been suggested in [13], with a unified presentation in [23]. In order to accelerate convergence, equations are modified in order to account for contributions from the Schur complement. The extended nonlinear case has been treated, e.g., in $[16,14]$.

Explicit coupling (or: loosely, weakly coupled schemes) suffer from severe time step restrictions, and are not considered here.

In passing we note that in the non-linear case, the linear system arising from a fully-implicit scheme can also be solved by a block-iterative preconditioner. This is similar to iterative coupling. However, since the problem at hand is linear, we do not formally distinguish these two cases here.

With respect to parallel performance, some investigations for an iteratively coupled scheme with implicit Euler time-stepping have been made, e.g., in $[11,23]$. In this work, we investigate a monolithic solver with two key components: The first component is an adaptive time-stepping that is is based on (linearly implicit) extrapolation methods [7]. The building block of this method are sequences of (linearly-) implicit Euler steps, that are combined with an Aitken-Neville extrapolation. The second component is a monolithic multi-level solver. It is inspired by works on scalable solvers for the Stokes system in $[9,12]$. Here, however, the Uzawa-type smoother, is constructed using the fixed stress approximation of the Schur complement [8].

The presentation is organized as follows: The mathematical model is formulated in Section 2, the description of the solver setup is given in Section 3. The work is concluded a numerical experiment in Section 4. 


\section{Governing equations}

In some domain $\Omega \subset \mathbb{R}^{d}$, we seek to solve the quasi-static Biot system for poroelasticity. The original formulation and derivation dates back to [2]; a recent description can be found, e.g., in the textbooks [5, 4]. The model is formulated in terms of a displacement $\mathbf{u}$ and a pressure $p$ :

$$
\begin{array}{r}
\partial_{t}\left(\frac{1}{M} p+\alpha \operatorname{div}(\mathbf{u})\right)+\nabla \cdot[\mathbf{q} \mid=0 \\
\nabla \cdot[\sigma]=-\mathbf{f} .
\end{array}
$$

These equations describe the conservation of mass and momentum of the fluid respectively. The system includes the Biot modulus $M$ and the Biot coeffient $0 \leq \alpha \leq 1$. We close the system by two constitutive material laws:

$$
\begin{aligned}
& \mathbf{q}=-\kappa(\nabla p-\rho \mathbf{g}) \\
& \sigma=\lambda \operatorname{div}(\mathbf{u}) \mathcal{I}+2 \mu \epsilon(\mathbf{u})-\alpha p \mathcal{I} .
\end{aligned}
$$

The Darcy velocity (1c) includes the permeability $\kappa$, and a density $\rho$, which is assumed to be constant. The linear elastic model (1d) for the stress tensor $\sigma$ includes the infinitesimal linearized strains $\epsilon(\mathbf{u})=\frac{1}{2}\left(\nabla \mathbf{u}+\nabla \mathbf{u}^{T}\right)$ and the Lamé parameters $\lambda, \mu$.

Relationship to engineering constants

The presented model includes the material parameters $\alpha, \lambda, \mu, M$, and $\kappa$. These can also be expressed in terms of the following engineering constants:

The parameter $\mu$ is the shear modulus which also sometimes denoted by $G$. The Lamé parameters $\lambda, \mu$ are related to other moduli as follows:

$$
\begin{aligned}
& \lambda=\frac{2 \nu G}{1-2 \nu}=K-\frac{2}{3} G=\frac{\nu E}{(1+\nu)(1-2 \nu)} \\
& \mu=G=\frac{3 K(1-2 \nu)}{2(1+\nu)}=\frac{E}{2(1+\nu)}
\end{aligned}
$$

Poisson's ratio is given by $\nu=\frac{\lambda}{2(\lambda+G)}$. Moreover, we can use the elasticity modulus $E$, and the bulk modulus $K=\lambda+\frac{2}{3} G$.

Following [4], we introduce $\xi=\frac{1}{M} p+\alpha e, e:=\operatorname{div}(u)$ i in (1a) as the variation of fluid content in a representative volume (relative to the original configuration). The undrained case corresponds to $\xi=0$, i.e. $p=-M \alpha e$. Inserting this into (1d) yields a redefined ( $p$ independent) stress tensor with parameters $\lambda_{u}=\lambda+M \alpha^{2}, K_{u}=K+M \alpha^{2}$, and $\nu_{u}=\frac{\lambda_{u}}{2\left(\lambda_{u}+G\right)}$. 
Variational form

In the variational form of (1), we seek to find $(p, \mathbf{u}) \in V_{p} \times V_{u}, V_{u} \subset L^{2}(\Omega)$, $V_{p} \subset H^{1}(\Omega)^{d}$ such that

$$
\begin{array}{r}
\int \epsilon(\mathbf{u}): \mathcal{C}: \epsilon(\mathbf{v})-\int \alpha \nabla \cdot \mathbf{v} p+\int \mathbf{f} \mathbf{v}=0 \\
\int \frac{1}{M} \dot{p} r+\int \alpha \nabla \cdot \dot{\mathbf{u}} r+\int \kappa \nabla p \nabla r-\int q r=0
\end{array}
$$

holds for all $(r, \mathbf{v}) \in V_{p} \times V_{u}$. Note that boundary conditions have been neglected for the ease of presentation. In the discrete setting, we replace $V_{p}$ and $V_{u}$ by discrete spaces $V_{p}^{h}$ and $V_{u}^{h}$. Here, we use conforming finite elements that are element-wise given by polynomials of degree 1 and 2 respectively.

Employing an implicit Euler scheme for time discretization, i.e.,

$$
\mathbf{u}_{t+\tau}:=\mathbf{u}_{t}^{h}+\triangle \mathbf{u}^{h}, p_{t+\tau}^{h}:=p_{t}^{h}+\triangle p^{h}
$$

results in a large linear system in the following block form:

$$
\left[\begin{array}{ll}
K_{11} & K_{12} \\
K_{21} & K_{22}
\end{array}\right]\left[\begin{array}{l}
\triangle p^{h} \\
\triangle \mathbf{u}^{h}
\end{array}\right]=-\left[\begin{array}{l}
r_{u}^{h} \\
r_{p}^{h}
\end{array}\right]
$$

The particular form of the right hand side is not important here. In the matrix

$$
\mathcal{K}:=\left[\begin{array}{ll}
K_{11} & K_{12} \\
K_{21} & K_{22}
\end{array}\right]:=\left[\begin{array}{cc}
A_{u u} & \alpha G_{u p} \\
-\alpha G_{u p}^{T} & \left(\frac{1}{M} M_{p p}+\tau \kappa A_{p p}\right)
\end{array}\right] .
$$

we identify the terms from (2) and note in passing, that the second diagonal term vanishes, if $\frac{1}{M} \rightarrow 0$ and $\tau \kappa \rightarrow 0$ simultaneously. In this case, standard smoothers are not applicable. In general (3) is a saddle point problem, as can, e.g., be seen by multiplying the second row by -1 and by using the abstract block decomposition:

$$
\mathcal{K}=\left[\begin{array}{cc}
I & \\
K_{21} K_{11}^{-1} & I
\end{array}\right]\left[\begin{array}{ll}
K_{11} & \\
& S_{22}
\end{array}\right]\left[\begin{array}{cc}
I & K_{11}^{-1} K_{12} \\
0 & I
\end{array}\right]
$$

with $S_{22}=K_{22}-K_{21} K_{11}^{-1} K_{12}$ denoting the Schur complement. This decomposition is also important for the iterative solvers that are introduced in the next section. 


\section{Algorithmic details}

\subsection{Iterative schemes}

Different strategies for solving (3) have been suggested in the literature. An overview on general saddle-point problems is given, e.g., in [1]. Most methods are based on iterative schemes of the following from

$$
x^{(k+1)}=x^{(k)}+\widetilde{\mathcal{K}}^{-1}\left(f-\mathcal{K} x^{(k)}\right) .
$$

where $\widetilde{\mathcal{K}}^{-1}$ is an approximate inverse that can be computed easily. In order to devise $\widetilde{\mathcal{K}}^{-1}$, one can employ the block decomposition (4) to define different block preconditioners, e.g.,

$$
\mathcal{W}_{l}=\left[\begin{array}{ll}
K_{11} & \\
K_{21} & K_{22}
\end{array}\right], \mathcal{W}_{d}=\left[\begin{array}{ll}
K_{11} & \\
& S_{22}
\end{array}\right], \mathcal{W}_{u}=\left[\begin{array}{rr}
K_{11} & K_{12} \\
& S_{22}
\end{array}\right]
$$

Inexact variants can also be devised, e.g.,

$$
\widetilde{\mathcal{W}}_{l}=\left[\begin{array}{ll}
\tilde{K}_{11} & \\
K_{21} & \tilde{S}_{22}
\end{array}\right], \widetilde{\mathcal{W}}_{d}=\left[\begin{array}{cc}
\tilde{K}_{11} & \\
& \tilde{S}_{22}
\end{array}\right], \widetilde{\mathcal{W}}_{u}=\left[\begin{array}{cc}
\tilde{K}_{11} & K_{12} \\
& \tilde{S}_{22}
\end{array}\right]
$$

It is well know, e.g. [3, 23], that the term $K_{21} K_{11}^{-1} K_{12}$ is spectrally equivalent to a rescaled mass matrix $M_{p p}$. Using the approximation

$$
S_{22}=K_{22}-K_{21} K_{11}^{-1} K_{12} \approx\left(\frac{1}{M}+\frac{\alpha^{2}}{K}\right) M_{p p}+\tau \kappa A_{p p}
$$

yields the fixed stress splitting [13]. We do not follow this approach here, but rely on the decomposition for building a smoother $[15,8]$ for a monolithic multigrid method.

\subsection{Monolithic multigrid solver}

Multigrid methods, as introduced in greater detail, e.g. in the monographs $[10,20]$, are based on the property that all frequencies in the error are reduces equally well. This is obtained by an efficient interplay between smoothing and interpolation between grid levels. These two components need to be defined:

The grid transfer operators are given by the standard operators as defined (elementwise) by the Lagrange polynomials. The coarse grid operators are recursively defined by the Galerkin products. For smoothing we employ an inxact Uzawa method, as suggested for Biot-System in [15, 8], with the 
following modifications: In the forward sweep, we use $\widetilde{\mathcal{W}}_{l}$, where the inverse of $\tilde{K}_{11}$ is approximated by a forward Gauss-Seidel sweep for $A_{u u}$, and the inverse of $\widetilde{S}_{22}$ is given by a damped Jacobi iteration for the right hand side of (5). The backward sweep is built using $\widetilde{\mathcal{W}}_{u}$ using the adjoint iterations. Note that a similar approach has successfully been used for the (related) Stokes equation before $[12,9]$ with excellent scalability properties.

\subsection{Software}

All algorithms and solvers have been implemented in the software framework UG4 $[22,18]$. Details of the MPI based parallelization of UG4's multigrid solver, highly scalable on hundreds of thousands of processes, have been described in previous reports, e.g. [19].

\section{Results}

\subsection{Benchmark problem}

As a benchmark problem, we consider the deformation of a cylinder, as suggested by de Leeuw [6, 4]: For $t>0$ a cylinder of radius $a$ is subject to uniform radial stress $\sigma_{r r}=-q$ at the cylinder wall, while drainage is instantaneously induced by $p=0$ outside.

This problem has an analytical solution [21]. Ibid., the initial value is given by a homogeneous pressure distribution corresponding to the undrained case for given $q$ :

$$
p(\mathbf{x}) \equiv p_{0}=\frac{M \alpha}{K+\frac{1}{3} G+M \alpha^{2}} q
$$

and its corresponding consistent displacement field $\mathbf{u}_{0}$. Note, that the first factor evaluates as 1, i.e., $p 0=q$, if solid and fluid are incompressible $(\alpha=1$, $M=\infty)$. The full set of parameters is provided in Tab. 1 .

Figure 1 shows the geometry as well as the solution after some time.

Figure 2 compares the analytical and numerical solution with respect to the normalized pressure $p\left(\mathbf{x}_{0}\right) / p_{0}$ at the center $\mathbf{x}_{0}$ of the cylinder. As $\nu<$ 0.5 the configuration gives rise to the well-known Mandel-Cryer effect: The pressure in the interior increases first, before it relaxes and decays to $p \rightarrow 0$. We also note that the adaptive time-stepping captures the dynamics correctly and selects the exponentially increasing time steps. In our case, the error was measured in the L2 norm for the pressure $p$, with a relative tolerance of $0.1 \%$. 


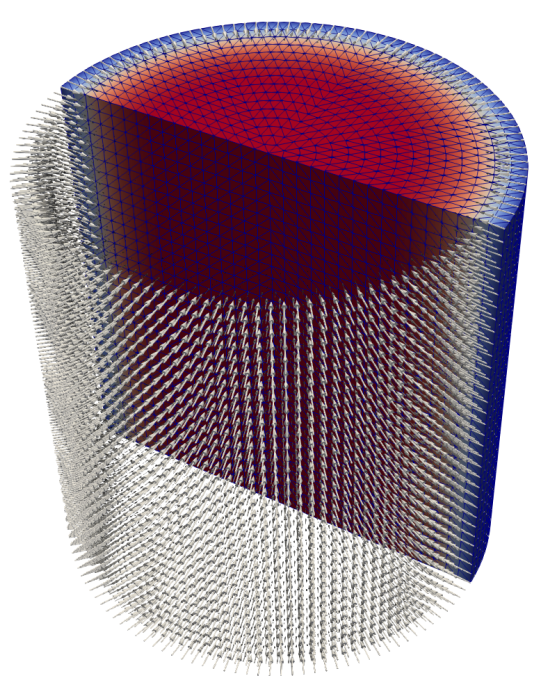

Fig. 1 Benchmark problem: Illustration of pressure and deformation.

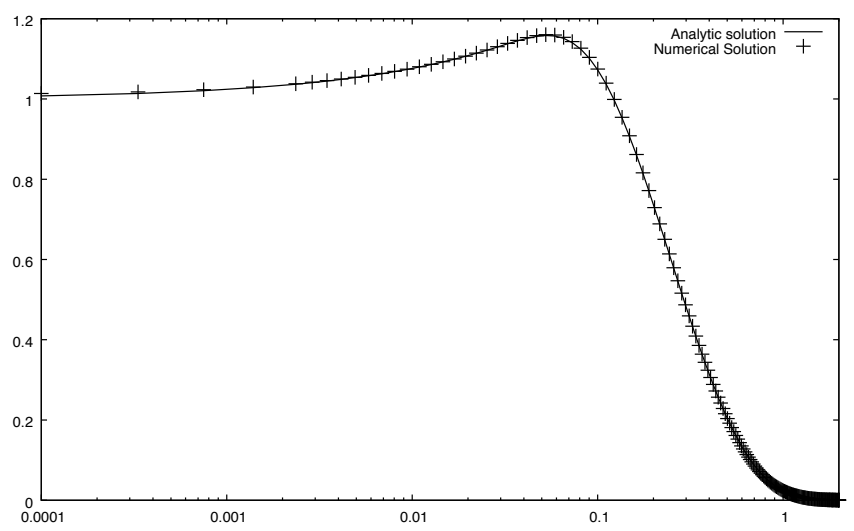

Fig. 2 Benchmark problem: Normalized pressure $p / p_{0}$ at the core of the cylinder as a function of the dimensionless time. The Mandel-Cryer effect leads to an increase in pressure that is followed by a drop. Note, that time the extrapolation scheme selects an exponentially increasing time step size. 


\begin{tabular}{cc}
\hline Parameter & Value Unit \\
\hline$E$ & $80 \mathrm{MPa}$ \\
$M$ & $1.1 \times 10^{7} \mathrm{MPa}$ \\
$\nu$ & 0.25 \\
$\alpha$ & 1 \\
$\kappa$ & $10^{-6} \mathrm{~m}^{2} /(\mathrm{Pa} \cdot \mathrm{s})$ \\
\hline$a$ & $1 \mathrm{~m}$ \\
$q$ & $1 \mathrm{MPa}$ \\
\hline
\end{tabular}

Table 1 Parameters for the test case. Although computations are perfomed in the adimensional form, they are provided with physical units here for the sake of orientation.

\subsection{Load balancing and grid distribution}

The performance of the multigrid algorithm depends on a proper load balancing. Following [19], the grid distribution quality $q_{l}$ onto $P_{l}$ processes on level $l$ of the hierarchy is computed as

$$
q_{l}:= \begin{cases}\frac{n_{l}^{\text {total }}-n_{l}^{\max }}{n_{l}^{\max } \cdot\left(P_{l}-1\right)}, & P_{l}>1 \\ 1, & P_{l}=1\end{cases}
$$

Here, $n_{l}^{p}$ is the number of elements in level $l$ on process $p$, and

$$
n_{l}^{\text {total }}:=\sum_{p=1}^{P_{l}} n_{l}^{p}, n_{l}^{\max }:=\max _{p=1, \ldots, P_{l}} n_{l}^{p} .
$$

The quality measure $q_{l}$ is in the range $[0,1]$. For $q_{l}=0$ all elements of level $l$ are contained on one process only, and $q_{l}=1$ indicates that the elements are shared equally among all processes.

\subsection{Numerical experiments}

The performance of the solver is investigated in a weak scaling study, using isotropic refinement. Problem size and involved number of cores increase as reported in Table 2:

\begin{tabular}{rcr}
\hline PEs & Levels $l$ & DoFs \\
96 & 5 & $6,714,692$ \\
768 & 6 & $53,070,468$ \\
6144 & 7 & $421,991,684$ \\
\hline
\end{tabular}

Table 2 Each line corresponds to an individual run. Recorded are the number of processes $(P E)$, the number of levels $(l)$, and the number of degrees of freedom (DoFs). 
Using a hierarchical load-balancing and distribution scheme [18], not all processes are contributing on all levels. For each configuration, Table 3 provides a summary of number of processes used on each level of the multigrid hierarchy. Table 4 shows the levelwise distribution qualities for each run.

\begin{tabular}{lllllllll}
\hline $\mathrm{PE}$ & $\mathrm{lv}-0$ & $\mathrm{lv}-1$ & $\mathrm{lv}-2$ & $\mathrm{lv}-3$ & $\mathrm{lv}-4$ & $\mathrm{lv}-5$ & $\mathrm{lv}-6$ & $\mathrm{lv}-7$ \\
\hline 96 & 1 & 1 & 96 & 96 & 96 & 96 & - & - \\
768 & 1 & 1 & 96 & 96 & 768 & 768 & 768 & - \\
6144 & 1 & 1 & 96 & 96 & 6144 & 6144 & 6144 & 6144 \\
\hline
\end{tabular}

Table 3 Number of processes used on each level for the individual runs

\begin{tabular}{lllllllll}
\hline $\mathrm{PE}$ & $\mathrm{lv}-0$ & $\mathrm{lv}-1$ & $\mathrm{lv}-2$ & $\mathrm{lv}-3$ & $\mathrm{lv}-4$ & $\mathrm{lv}-5$ & $\mathrm{lv}-6$ & $\mathrm{lv}-7$ \\
\hline 96 & 1 & 1 & 1 & 1 & 0.82 & 0.82 & - & - \\
768 & 1 & 1 & 1 & 1 & 0.84 & 0.84 & 0.99 & - \\
6144 & 1 & 1 & 1 & 1 & $\mathbf{0 . 4 0}$ & $\mathbf{0 . 4 0}$ & 0.90 & 0.90 \\
\hline
\end{tabular}

Table 4 Distribution qualities for each level of the multigrid hierarchy for the different runs

Aiming for representative results, performance has been evaluated in an interval of $1 h$ wall clock time, which corresponds to at least 25 time steps. One time step corresponds is computed by extrapolation [7], and is based of three implicit Euler steps. In each of these steps, the resulting system has been solved using a BiCGStab algorithm, that was preconditioned by the monolithic multigrid solver described in Section 3. The residual were reduced by 8 orders of magnitude, or, alternatively, to absolute tolerance of $10^{-14}$.

Figure 3 provides the results for this setup: As a performance measure, we consider the average number of time steps per wall clock time unit. Results range between 0.5 steps / $\mathrm{min}$ in the worst case (W-cycle on $6144 \mathrm{PE}$ ) and 0.8 steps $/ \mathrm{min}$ in the best case (F-cycle on $96 \mathrm{PE}$ ). On $96 \mathrm{PE}, \mathrm{W}$-cycle and Fcycle show almost identical performance. However, as can be expected, the $\mathrm{W}$ cycle deteriorates when increasing the level of parallelism. This issue is well known. It is due to increasing number of smoother and coarse grid correction calls on the coarse levels $l$, which is a accompanied by a relative reduction of the level of parallelism (as illustrated in Table 3). Using the F cycle mitigates these issues, and results improve slightly with $\approx 0.7 \mathrm{steps} / \mathrm{min}$ on $768 \mathrm{PE}$, and $\approx 0.6$ steps $/ \mathrm{min}$ on $6144 \mathrm{PE}$ respectively. Using the $\mathrm{V}$-cycle is desirable from the point of view of scalability, but is not a viable alternative here. 


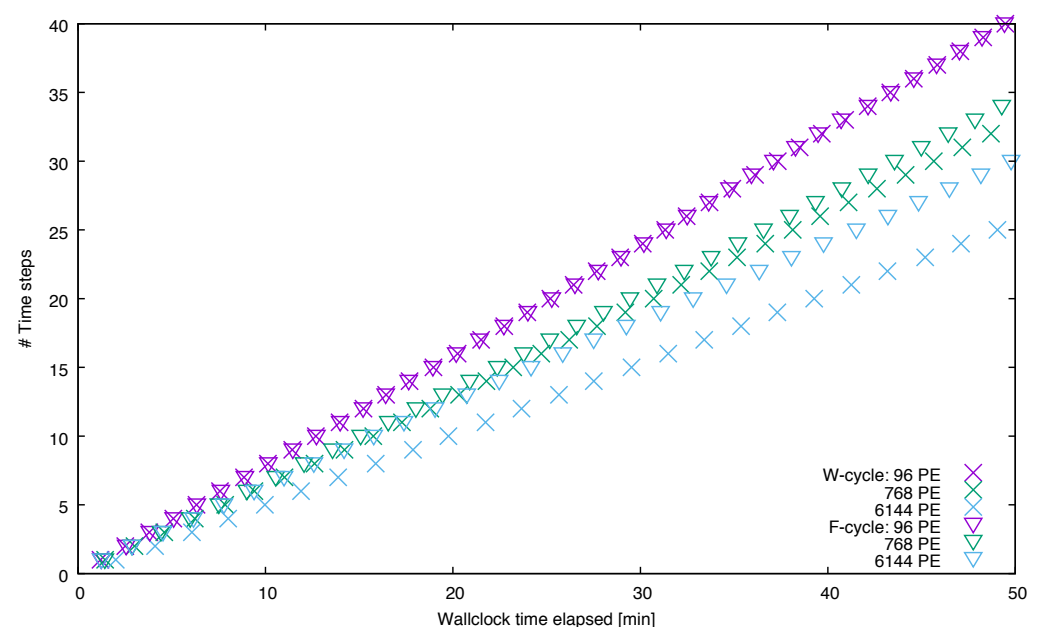

Fig. 3 Time steps per elapsed wall clock time: Results of weak scaling for 96, 768, and $6144 \mathrm{PE}$ for W- and F-cycle respectively.

\section{Conclusion}

The presented preliminary results show the potential of the combination of time-stepping and linear solver. Using an adaptive time integrator is key for improving the performance. The linear solver indicates its potential, but improving its scalability porperties is still subject to further investigations and profiling. Although for the F-cycle a relative efficiency of at least $75 \%$ is obtained, it must be clarified, if this can be improved further. The imbalance shown in Table 4 (row for $6144 \mathrm{PE}$ ) may have a severe impact.

\section{Acknowledgement}

This work has been supported by the DFG in the German Priority Programme 1648 - Software for Exascale Computing in the project Exasolvers (WI 1037/24-2). The authors would like to thank Sebastian Reiter and Michael Lampe for support for generating the geometries and discussions on scalability aspects of Hazel Hen. Moreover, the technical support by HLRS staff is gratefully acknowledged. 


\section{References}

1. M. Benzi, G. H. Golub, and J. Liesen. Numerical solution of saddle point problems. Acta numerica, 14:1-137, 2005.

2. M. A. Biot. General theory of threedimensional consolidation. Journal of Applied Physics, 12(2):155164, 1941.

3. N. Castelletto, J. White, and H. Tchelepi. Accuracy and convergence properties of the fixed-stress iterative solution of two-way coupled poromechanics. International Journal for Numerical and Analytical Methods in Geomechanics, 39(14):1593-1618, 2015.

4. A. H.-D. Cheng. Poroelasticity, volume 27 of Theory and Applications of Transport in Porous Media. Springer International Publishing Switzerland, 2016.

5. O. Coussy. Poromechanics. John Wiley \& Sons, Ltd, 2004.

6. E. de Leeuw. The theory of three-dimensional consolidation applied to cylindrical bodies. In Proceedings of 6th international conference on soil mechanics and foundation engineering, Montreal, volume 1, pages 287-290, 1965.

7. P. Deuflhard. One-step and Extrapolation Methods for Differential-Algebraic Systems. Numerische Mathematik, 51:501-516, 1987.

8. F. J. Gaspar and C. Rodrigo. On the fixed-stress split scheme as smoother in multigrid methods for coupling flow and geomechanics. Computer Methods in Applied Mechanics and Engineering, 326:526-540, Nov. 2017.

9. B. Gmeiner, M. Huber, L. John, U. Rüde, and B. Wohlmuth. A quantitative performance study for stokes solvers at the extreme scale. Journal of Computational Science, 17:509-521, 2016.

10. W. Hackbusch. Multi-Grid Methods and Applications. Springer, Berlin, 1985.

11. C. J. B. Haga. Numerical methods for basin-scale poroelastic modelling. PhD thesis, University of Oslo, 2011.

12. L. John, U. Rüde, B. Wohlmuth, and W. Zulehner. On the analysis of block smoothers for saddle point problems. ArXiv e-prints, Dec. 2016.

13. J. Kim, H. Tchelepi, and R. Juanes. Stability, accuracy, and efficiency of sequential methods for coupled flow and geomechanics. SPE Journal, 16(2), Jun 2011.

14. S. Lee, M. F. Wheeler, and T. Wick. Iterative coupling of flow, geomechanics and adaptive phase-field fracture including level-set crack width approaches. J. Comput. Appl. Math., 314(C):40-60, Apr. 2017.

15. P. Luo, C. Rodrigo, F. J. Gaspar, and C. W. Oosterlee. Uzawa smoother in multigrid for the coupled porous medium and stokes flow system. SIAM Journal on Scientific Computing, 39(5):633-661, 2017.

16. A. Mikelić, B. Wang, and M. F. Wheeler. Numerical convergence study of iterative coupling for coupled flow and geomechanics. Computational Geosciences, 18(3):325341, Aug 2014.

17. A. Mikelić and M. F. Wheeler. Convergence of iterative coupling for coupled flow and geomechanics. Computational Geosciences, 17(3):455-461, Jun 2013.

18. S. Reiter, A. Vogel, I. Heppner, M. Rupp, and G. Wittum. A massively parallel geometric multigrid solver on hierarchically distributed grids. Comp. Vis. Sci., 16(4):151-164, 2013.

19. S. Reiter, A. Vogel, A. Nägel, and G. Wittum. A massively parallel multigrid method with level dependent smoothers for problems with high anisotropies. High Performance Computing in Science and Engineering 16., pages 667-675, 2016.

20. U. Trottenberg, C. W. Oosterlee, and A. Schüller. Multigrid. Academic Press, San Diego, CA, 2001. contributions by A. Brandt and P. Oswald and K. Stüben.

21. A. Verruijt. Theory and problems of poroelasticity. Delft University of Technology, 2013.

22. A. Vogel, S. Reiter, M. Rupp, A. Nägel, and G. Wittum. UG 4: A novel flexible software system for simulating PDE based models on high performance computers. Comp. Vis. Sci., 16(4):165-179, 2013. 
23. J. A. White, N. Castelletto, and H. A. Tchelepi. Block-partitioned solvers for coupled poromechanics: A unified framework. Computer Methods in Applied Mechanics and Engineering, 303:55-74, 2016. 